\title{
Gestational Ovarian Choriocarcinoma
}

National Cancer Institute

\section{Source}

National Cancer Institute. Gestational Ovarian Choriocarcinoma. NCI Thesaurus. Code C40442.

A rare malignant trophoblastic tumor that arises from the ovary as a result of ectopic ovarian pregnancy. There is no germ cell component present. 